\title{
Tormenta tiroidea: a propósito de un caso
}

\section{Thyroid storm: a case report}

Pág. 2, 8

Recibido: 04-06-2020

Aceptado: 24-08-2020

Dra. Rebeca Gutiérrez Navarro ${ }^{1}$

Dr. Christian García Quirós²

1. Médico General,Trabajador independiente, San José, Costa Rica.

2. Especialista en Medicina Interna. Hospital San Juan de Dios, Caja Costarricense del Seguro Social.

\section{RESUMEN}

Masculino de 56 años, hipertenso, neumópata crónico e hipertiroideo con pobre adherencia al tratamiento, que presenta Tormenta Tiroidea, en el contexto de fractura de cadera traumática como factor desencadenante.

\section{PALABRAS CLAVE}

emergencia endocrinológica, tormenta tiroidea, tirotoxicosis, fallo multiorgánico.

\section{ABSTRACT}

A 56-year-old male, hypertensive, with chronic lung disease and hyperthyroid with inadequate adherence, presents with thyroid storm as a complication of traumatic hip fracture.

\section{KEY WORDS}

endocrinological emergency, thyroid storm, thyrotoxicosis, multiorgan failure.

\section{CASO CLÍNICO}

Al llamado de una valoración preoperatoria, se encuentra un masculino de 56 años, tabaquista, hipertenso en tratamiento con enalapril $5 \mathrm{mg} /$ día, hidroclorotiazida $25 \mathrm{mg} / \mathrm{día}$, portador de neumopatía crónica no estratificada sin tratamiento e hipertiroidismo, aproximadamente 2 años de evolución con pobre adherencia al tratamiento.

El paciente cuenta con historia de pérdida de peso significativa en los últimos 10 meses (aproximadamente $>20 \mathrm{~kg}$ ), no intencional, de etiología desconocida, e ingresa por fractura transtrocantérica de fémur derecho, debido a trauma producido por caída desde su propia altura.

A la exploración se encuentra a un paciente crónicamente enfermo, caquéctico, en quién se documenta durante valoración preoperatoria: presión arterial en 199/126 mmHg, frecuencia cardíaca de 237 latidos por minuto, frecuencia respiratoria de 41 respiraciones por minuto, 
saturación de oxígeno al 91\% (FiO2 21\%), temperatura $39.8^{\circ} \mathrm{C}$ y una glicemia de $49 \mathrm{mg} /$ $\mathrm{dL}$. Se encontraba conciente, vigil, agitado y combativo; con fluctuación del curso del pensamiento, este desorganizado, inatento, sin obedecerórdenes. Exoftalmos presente, bilateral acompañado de midriasis pupilar bilateral. Taquipneico, con uso de musculatura accesoria de la respiración, se auscultó murmullo vesicular bilateral conservado sin estertores audibles. Los ruidos cardiacos eran disrrítmicos, taquicárdicos de buen tono, sin soplos cardiacos aubibles. En miembros superiores llamó la atención tremor distal. Sin otros hallazgos patológicos relevantes.

Cabe hacer mención que no se evidenciaba a la exploración física inicial aumento de volumen de glándula tiroidea ni soplos en cuello. Los laboratorios fueron tomados el mismo día de la valoración, y arrojaron los siguientes resultados: hemoglobina en $7.9 \mathrm{~g} / \mathrm{dL}$, hematocrito en $23 \%$, plaquetas $110000 \mathrm{mil} / \mathrm{mm}^{3}$, leucocitos en $3800 \mathrm{mil} / \mathrm{mm}^{3}$, con un diferencial de $2660 \mathrm{mil} /$ $\mathrm{mm}^{3}$ polimorfonucleares $(70 \%)$ y $950 \mathrm{mil} / \mathrm{mm}^{3}$ linfocitos (25\%), nitrogeno ureico (BUN) en 8 $\mathrm{mg} / \mathrm{dL}$ y creatinina (creat) $0.23 \mathrm{mg} / \mathrm{dL}$, con una relación BUN/creat de 34, lo cual demostraba retención de compuestos nitrogenados. El perfil electrolítico mostró hipocalcemia (calcio $6.7 \mathrm{mg} /$ dl [corregido $7.9 \mathrm{mg} / \mathrm{dL}$ ]), sin otras alteraciones, las enzimas hepáticas AST en $65 \mathrm{U} / \mathrm{L}, \mathrm{ALT} \mathrm{U} / \mathrm{L}$ en 55 , GGT $89 \mathrm{U} / \mathrm{L}$ y una fosfatasa alcalina en $145 \mathrm{U} / \mathrm{L}$, la albúmina en $2 \mathrm{~g} / \mathrm{dL}$. Y la bioquímica tiroidea reportó una TSH claramente suprimida $<0.005 \mathrm{mU} / \mathrm{L}$ con aumento de T4 y T3 en 7 $\mathrm{ng} / \mathrm{dl}$ y, $13.8 \mathrm{ng} / \mathrm{dl}$ respectivamente. El INR se encontró en 3.0 y los gases arteriales mostraron una alcalosis metabólica con hiperlactatemia

El electrocardiograma evidenció una Fibrilación auricular de alta respuesta ventricular inestable
(Fig.1). La radiografía de tórax mostró una zona de infiltrado algodonoso a nivel $1 / 3$ medio hemitórax derecho, reforzamiento hiliar izquierdo, sin otros hallazgos (Fig.2).

Debido a la inminente claudicación ventilatoria, se procedió a realizar secuencia rápida de intubación para proteger la vía aérea.

En este contexto clínico, asociado a historia de hipertiroidismo sin control ni tratamiento adecuados, se encontra frente a la sospecha clínica de una tormenta tiroidea. Se comentó el caso con la Unidad de Cuidados Intensivos para su estrecha vigilancia y se inició tratamiento supresor de hormona tiroidea con propiltiuracilo $200 \mathrm{mg} 4$ veces al día vo. El estado hiperadrenérgico se manejó con propranolol $1 \mathrm{mg}$ IV cada 15 minutos, para mantener una frecuencia cardíaca en $<100$ latidos por minuto y posteriormente se realizó el traslape con Propranolol oral $40 \mathrm{mg}$ cada $8 \mathrm{~h}$ VO.

Los líquidos se manejaron con dextrosa al $50 \% 100 \mathrm{cc}$ iv y suero fisiológico $0.9 \% 1$ litro IV alternado con $1 \mathrm{~L}$ solución mixta cada $8 \mathrm{~h}$. Se asoció gluconato de calcio 2 ampollas IV cada 8 horas. Se administró Lugol 10 gotas vo STAT y se continuó cada 6 horas.

Hidrocortisona 200 mg IV y se continuó con $50 \mathrm{mg}$ cada 6 horas IV. Cefotaxime $2 \mathrm{~g}$ iv stat y se continuó con $2 \mathrm{~g}$ cada $8 \mathrm{~h}$ iv asociado a Clindamicina $600 \mathrm{mg}$ cada $6 \mathrm{~h}$ por 7 días para tratarlaneumonía poraspiración detectada, como consecuencia de una posible broncoaspiración alimentaria debida a la alteración del estado de conciencia del paciente en días previos a su ingreso. 


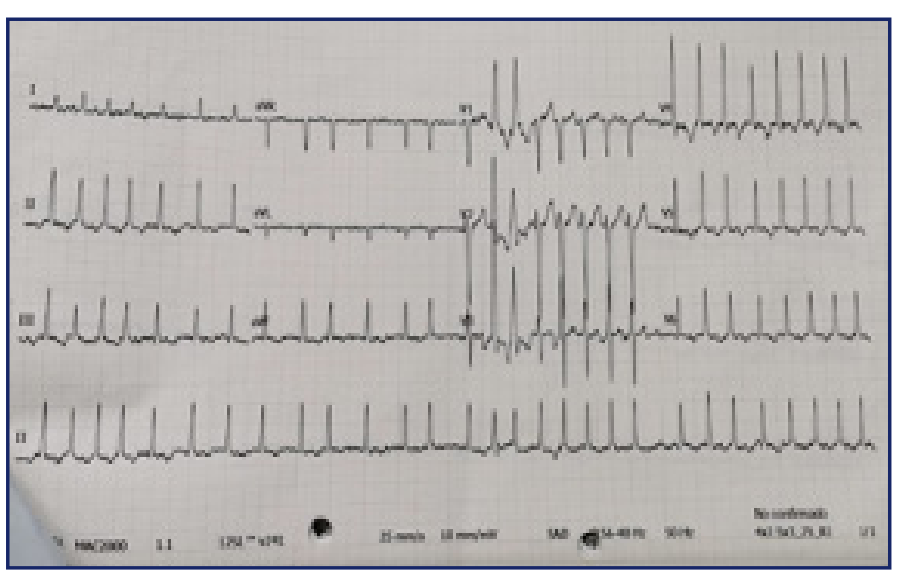

Figura 1. Fibrilación auricular de alta respuesta ventricular inestable.

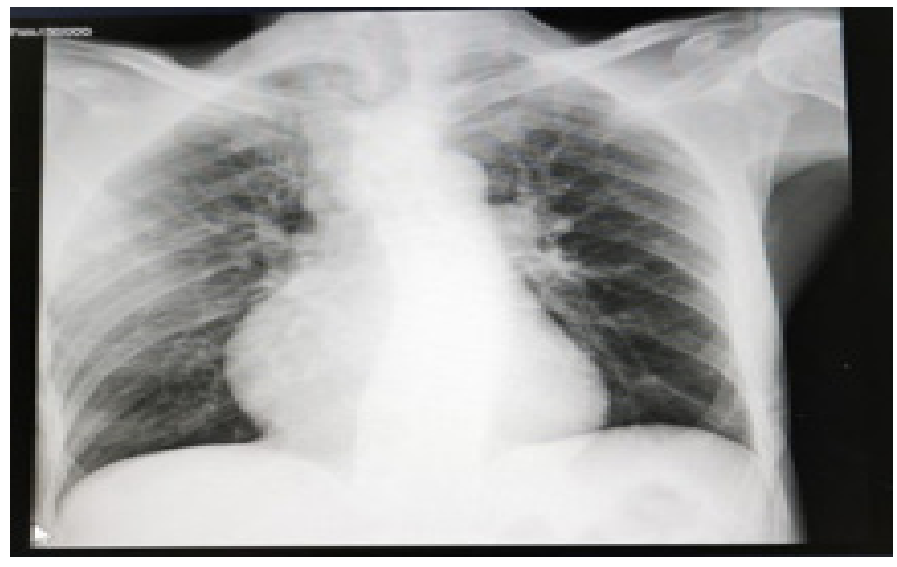

Figura 2. zona de infiltrado algodonoso a nivel $1 / 3$ medio hemitórax derecho, reforzamiento hiliar

\section{DISCUSIÓN Y ANÁLISIS DEL CASO}

La tormenta tiroidea es una complicación poco frecuente de estados hipertiroideos 0 tirotoxicosis, sin embargo, se trata de una emergencia endocrinológica que suele pasarse por alto, cuyo manejo y abordaje debe ser precoz.

Los pacientes se presentan con clínica aguda exacerbada de signos y síntomas clásicos de la tirotoxicosis (tabla I.). Su mortalidad ronda el $10-30 \%(2)$ y es debida al fallo multiorgánico como resultado del colapso de los mecanismos compensatorios(1).

Los pacientes con estados hipertiroideos previamente instaurados suponen un factor de riesgo para el desarrollo de la tormenta tiroidea, si se encuentran ante condiciones precipitantes como lo son: infecciones (más comúnmente del tracto respiratorio), traumas, cirugías, accidente cerebrovascular; así mismo como la tirotoxicosis no tratada o parcialmente tratada, la interrupción abrupta de medicamentos antitiroideos, y causas iatrogénicas. La etiología más común es la enfermedad de Graves, siendo el precipitante más común la infección. En el contexto de este paciente se encontra frente a dos factores precipitantes: el trauma de cadera que ocasionó fractura y la el proceso neumónico por broncoaspiración.

\begin{tabular}{|c|}
\hline Fiebre $>38^{\circ} \mathrm{C}$ \\
\hline Sistema nervioso central \\
\hline Inquietud \\
\hline Delirio \\
\hline Psicosis \\
\hline Somnolencia, letargo \\
\hline Coma \\
\hline Sistema Cardiovascular \\
\hline Taquicardia 130 lpm o >130 lpm en fibrilación auricular \\
\hline $\begin{array}{l}\text { Insuficiencia cardiaca congestiva: edema pulmunar, créditos, shock } \\
\text { cardiogénico o Clase IV NYHA o Clase III Killip. }\end{array}$ \\
\hline Sistema gastrointestinal / hepático \\
\hline Nauseas, Vómitos, diarrea \\
\hline Bilirrubina total $>3.0 \mathrm{mg} / \mathrm{dL}$ \\
\hline
\end{tabular}

Tabla I. Manifestaciones clínicas de Tormenta Tiroidea 
Los resultados de las hormonas tiroideas no son un parámetro indicativo de tormenta tiroidea, ya que no existen diferencias claras entre un paciente en tirotoxicosis y de otro con crisis tiroidea(1).

Dentro de los exámenes de laboratorio es posible encontrar leucocitosis o leucopenia, aumento de las enzimas hepáticas, hiperglucemia por inhibición de la liberación de insulina inducida por catecolaminas, hipercalcemia, elevación de la fosfatasa alcalina por hemoconcentración y estimulación de la resorción ósea mediada por hormona tiroidea(2).

En el contexto del paciente en estudio, la hipoglicemia podría deberse al hipercatabolisismo agudo con pobres reservas de glucógeno, así como la hipocalcemia leve en fases precoces de la Tormenrta Tiroidea que estimularán posteriormente la PTH para aumento de resorción ósea e hipercalcemia concomitante.

Hallazgos en el electrocardiograma pueden mostrar taquicardia sinusal o fibrilación auricular. Es importante la sospecha clínica precoz ante un posible escenario de tormenta tiroidea $y$, aunque actualmente no existe un consenso de criterios diagnósitcos, a modo de apoyo, suele utilizarse la escala de puntos de Burch y Wartofsky que va de los 0 a 140 puntos y según el puntaje obtenido, $<25$ puntos se interpreta como "improbabilidad de presentar tormenta tiroidea. Entre 25-44 puntos "tormenta tiroidea inminente" $y \geq 45$ puntos es "altamente sugestivo de tormenta tiroidea". En el el presente caso, el paciente contabilizó 80 puntos de la Escala BWPS compatible con Tormenta Tiroidea.

\begin{tabular}{|c|c|c|c|}
\hline \multicolumn{4}{|c|}{$\begin{array}{l}\text { TABLE } 2 \\
\text { Burch-Wartofsky Point Scale }\end{array}$} \\
\hline \multicolumn{2}{|l|}{ Temperature ( $\left.{ }^{\circ} \mathrm{F}\right)$} & \multicolumn{2}{|c|}{ Cardiovascular dysfunction } \\
\hline $99-99.9$ & 5 points & Tachycardia (beats/min) & \\
\hline $100-100.9$ & 10 & $99-109$ & 5 \\
\hline $101-101.9$ & 15 & $110-119$ & 10 \\
\hline $102-102.9$ & 20 & $120-129$ & 15 \\
\hline $103-103.9$ & 25 & $130-139$ & 20 \\
\hline$\geq 104.0$ & 30 & $\geq 140$ & 25 \\
\hline \multicolumn{2}{|c|}{ Central nervous system effects } & Atrial fibrillation & 10 \\
\hline Absent & 0 & \multicolumn{2}{|l|}{ Heart failure } \\
\hline Mild (agitation) & 10 & Mild (pedal edema) & 5 \\
\hline $\begin{array}{l}\text { Moderate (delirium, } \\
\text { psychosis, extreme lethargy) }\end{array}$ & 20 & Moderate (bibasilar rales) & 10 \\
\hline Severe (seizure, coma) & 30 & Severe (pulmonary edema) & 15 \\
\hline \multicolumn{2}{|c|}{ Gastrointestinal-hepatic dysfunction } & \multicolumn{2}{|l|}{ Precipitant history } \\
\hline \multirow{3}{*}{$\begin{array}{l}\text { Moderate (diarrhea, nausea/ } \\
\text { vomiting, abdominal pain) } \\
\text { Severe (unexplained jaundice) }\end{array}$} & & Positive & 0 \\
\hline & 10 & Negative & 10 \\
\hline & 20 & & \\
\hline \multicolumn{4}{|c|}{ Total: $<25$, storm unlikely; $25-45$, impending storm; > 45, thyroid storm } \\
\hline \multicolumn{4}{|c|}{ Source: Burch, Wartofsky. Endocrinol Metab Clin North Am. 1993.2 } \\
\hline
\end{tabular}

La Asociación Americana de Endocrinólogos Clínicos y la Asociación Americana de Tiroides recomiendan en sus guías el tratamiento específico, que inicia con el bloqueo definitivo de la síntesis de hormonas tiroideas con fármacos antitiroideos en dosis elevadas. El propiltiuracilo bloquea la síntesis de nueva hormona tiroidea, se administra una dosis de carga de $500-1000 \mathrm{mg}$ seguido de $250 \mathrm{mg}$ cada 4 horas (puede darse intravenoso).

El metamizol bloquea la nueva síntesis de hormona tiroidea y la conversión periférica de T4 a T3, se administra en dosis de 60$80 \mathrm{mg}$ cada día. Los betabloqueadores deben darse en conjunto para el control de signos y síntomas del estado adrenérgico aumentado. El propranolol a dosis de $60-80 \mathrm{mg}$ cada 4 horas. En dosis altas funciona como bloqueador, no solo beta adrenérgico, sino también de conversión periférica de la T4 a T3. 
El yodo (en presentaciones como yoduro de sodio, ipodato sódico, yoduro potásico o solución de Lugol) bloquea la liberación de hormonas tiroideas desde la glándula, sin embargo, debe tomarse en cuenta que este debe utilizarse una hora después del inicio de los antitiroideos.

Los glucocorticoides se utilizan a razón del bloqueo de la conversión periférica de T4 a T3 y como profilaxis contra la insuficiencia adrenal relativa. Suele administrarse hidrocortisona con $300 \mathrm{mg}$ IV dosis de carga, más $100 \mathrm{mg}$ cada 8 horas(3).

Como tratamiento definitivo, debe considerarse la cirugía o la ablación de la glándula tiroides para evitar futuras crisis tiroideas.

En nuestro contexto, es indudable que la cirugía se debió aplazar hasta lograr tener un control hormonal del eje tiroideo, el cual se logró al cabo de los 10 días posterior al inicio de medicamentos antitiroideos, con el posterior reemplazo de cadera, motivo de consulta inicial, con excelente evolución clínica de la emergencia endocrinológica, así como su egreso hospitalario ocurrió sin complicaciones mayores y fue seguido por control ambulatorio por el servicio de Endocrinología.

\section{CONCLUSIONES}

La Tormenta Tiroidea constituye la complicación más aparatosa de la tirotoxicosis, la cual puede desencadenarse en el contexto de procesos infecciosos, traumas, trasgresión aguda o abandono de medicamentos antitiroideos, esto como las causas mas frecuentes, cuyo reconocimiento del cuadro se debe al cumplimiento de los criterios de Burch y Wartofsky, cuyo puntaje debe ser mayor de 25 puntos como altamente probable o mayor de 45 como altamente sugestivo.

El objetivo del tratamiento es, no solo el control del eje tiroideo por medio de medicamentos antiroideos como el Lugol, betabloqueadores, PTU, sino controlar la noxa precipitante del caos hormonal que el paciente presenta, todo esto bajo vigilancia estricta de una unidad de cuidado intensivo.

El reconocimiento, abordaje y tratamiento precoz, tanto de la tormenta tiroidea, como su desencadenante, tiene una relación directamente proporcional en la sobrevida de los pacientes que lo presentan.

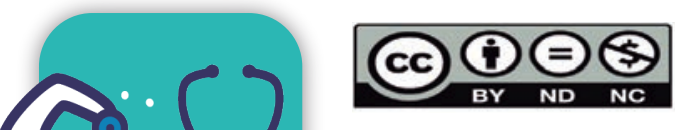




\section{BIBLIOGRAFÍA}

1. Akamizu T. (2018). Thyroid Storm: A Japanese Perspective. Thyroid : official journal of the American Thyroid Association, 28(1), 32-40. https://doi.org/10.1089/thy.2017.0243

2. Leal Curí, Lisette. (2012). Tormenta tiroidea. Revista Cubana de Endocrinología, 23(3), 264272. Recuperado en 27 de mayo de 2020, de http://scielo.sld.cu/scielo.php?script=sci_arttext\&pi$\mathrm{d}=$ S1561-29532012000300012\&lng=es\&tlng=es.

3. Bahn RS, Burch HB, Cooper DS, et al. Hyperthyroidism and other causes of thyrotoxicosis: management guidelines of the American Thyroid Association and American Association of Clinical Endocrinologists [published correction appears in Endocr Pract. 2013 Mar-Apr;19(2):384]. Endocr Pract. 2011;17(3):456520. doi:10.4158/ep.17.3.456

4. Ramírez Martínez, M., Gutiérrez Macías, A., de la Guerra Acebal, C., Lizarraga Zufiaurre, A., Bóveda Romeo, J., \& de la Villa, F. Crisis tirotóxica (tormenta tiroidea). A propósito de dos observaciones. Gaceta Médica de Bilbao. 2010; 107(2):64-67

5. Idrose A. M. (2015). Acute and emergency care for thyrotoxicosis and thyroid storm. Acute medicine \& surgery, 2(3), 147-157. https://doi.org/10.1002/ams2.104

6. Liang, C. M., Ho, M. H., Wu, X. Y., Hong, Z. J., Hsu, S. D., \& Chen, C. J. (2015). Thyroid storm following trauma: a pitfall in the emergency department. Injury, 46(1), 169-171. https://doi.org/10.1016/j. injury.2014.08.003

7. Ylli, D., Klubo-Gwiezdzinska, J., \& Wartofsky, L. (2019). Thyroid emergencies. Polish archives of internal medicine, 129(7-8), 526-534. https://doi.org/10.20452/pamw.14876

8. Wilkinson JN. Thyroid storm in a polytrauma patient. Anaesthesia. 2008;63(9):1001-1005. doi:10.1111/ j.1365-2044.2008.05541.x

9. Ross, D. S., Burch, H. B., Cooper, D. S., Greenlee, M. C., Laurberg, P., Maia, A. L., Rivkees, S. A., Samuels, M., Sosa, J. A., Stan, M. N., \& Walter, M. A. (2016). 2016 American Thyroid Association Guidelines for Diagnosis and Management of Hyperthyroidism and Other Causes of Thyrotoxicosis. Thyroid : official journal of the American Thyroid Association, 26(10), 1343-1421. https://doi.org/10.1089/thy.2016.0229

10. Burch H. B. (2019). Drug Effects on the Thyroid. The New England journal of medicine, 381(8), 749-761. https://doi.org/10.1056/NEJMra1901214

11. Banerjee, A., Bala, R., \& Aggarwal, R. (2019). Atypical presentation of thyroid storm: a diagnostic dilemma. BMJ case reports, 12(8), e231090. https://doi.org/10.1136/bcr-2019-231090 
12. Pangtey, G. S., Baruah, U., Baruah, M. P., \& Bhagat, S. (2017). Thyroid Emergencies: New Insight into Old Problems. The Journal of the Association of Physicians of India, 65(8), 68-76.

13. Bourcier, S., Coutrot, M., Kimmoun, et al (2020). Thyroid Storm in the ICU: A Retrospective Multicenter Study. Critical care medicine, 48(1), 83-90. https://doi.org/10.1097/CCM.0000000000004078

14. Baena, J. C., Padilla, J., \& Guzmán, G. (2017). Tormenta tiroidea asociada a disfunción multiorgánica [Thyroid storm associated with multiorganic dysfunction]. Medicina, 77(4), 337-340.

15. Jacobi J. (2019). Management of Endocrine Emergencies in the ICU. Journal of pharmacy practice, 32(3), 314-326. https://doi.org/10.1177/0897190019834771

16. Ishii M. (2017). Endocrine Emergencies With Neurologic Manifestations. Continuum (Minneapolis, Minn.), 23(3, Neurology of Systemic Disease), 778-801. https://doi.org/10.1212/CON.0000000000000467

17. Idrose A. M. (2015). Acute and emergency care for thyrotoxicosis and thyroid storm. Acute medicine \& surgery, 2(3), 147-157. https://doi.org/10.1002/ams2.104

18. Swee, d., Ching, C. L., \& Lim, A. (2015). Clinical characteristics and outcome of thyroid storm: a case series and review of neuropsychiatric derangements in thyrotoxicosis. Endocrine practice : official journal of the American College of Endocrinology and the American Association of Clinical Endocrinologists, 21(2), 182-189. https://doi.org/10.4158/EP14023.OR

19. Sharp, C. S., Wilson, M. P., \& Nordstrom, K. (2016). Psychiatric Emergencies for Clinicians: The Emergency Department Management of Thyroid Storm. The Journal of emergency medicine, 51(2), $155-158$. https://doi.org/10.1016/j.jemermed.2016.01.032

20. Bacuzzi, A., Dionigi, G., Guzzetti, L., De Martino, A. I., Severgnini, P., \& Cuffari, S. (2017). Predictive features associated with thyrotoxic storm and management. Gland surgery, 6(5), 546-551. https://doi. org/10.21037/gs.2017.07.01

21. Ikeoka, T., Otsuka, H., Fujita, N., Masuda, Y., Maeda, S., Horie, I., Ando, T., Abiru, N., \& Kawakami, A. (2017). Thyroid Storm Precipitated by Diabetic Ketoacidosis and Influenza A: A Case Report and Literature Review. Internal medicine (Tokio, Japan), 56(2), 181-185. https://doi.org/10.2169/internalmedicine.56.7593 